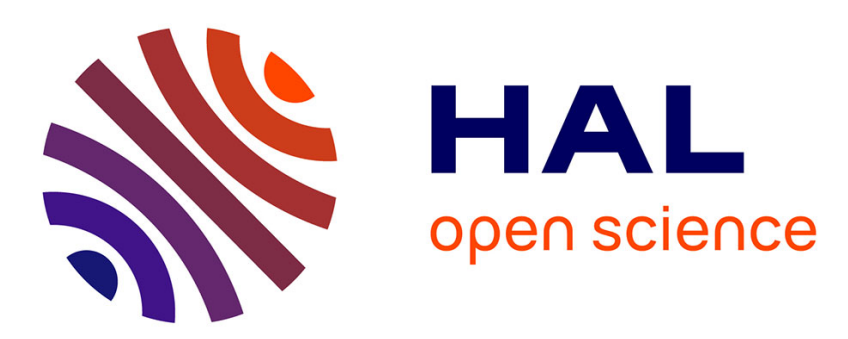

\title{
Étalonnage en luminance spectrale énergétique dans l'ultraviolet à l'aide d'un plasma d'hydrogène

\author{
P. Fieffe-Prevost
}

\section{To cite this version:}

P. Fieffe-Prevost. Étalonnage en luminance spectrale énergétique dans l'ultraviolet à l'aide d'un plasma d'hydrogène. Revue de Physique Appliquée, 1977, 12 (9), pp.1243-1246. 10.1051/rphysap:019770012090124300 . jpa-00244305

\section{HAL Id: jpa-00244305 https://hal.science/jpa-00244305}

Submitted on 1 Jan 1977

HAL is a multi-disciplinary open access archive for the deposit and dissemination of scientific research documents, whether they are published or not. The documents may come from teaching and research institutions in France or abroad, or from public or private research centers.
L'archive ouverte pluridisciplinaire HAL, est destinée au dépôt et à la diffusion de documents scientifiques de niveau recherche, publiés ou non, émanant des établissements d'enseignement et de recherche français ou étrangers, des laboratoires publics ou privés. 


\title{
ÉTALONNAGE EN LUMINANCE SPECTRALE ÉNERGÉTIQUE DANS L'ULTRAVIOLET A L'AIDE D'UN PLASMA D'HYDROGẼNE (*)
}

\author{
P. FIEFFE-PREVOST
}

\author{
Institut National de Métrologie, C. N. A. M., 292, rue Saint-Martin, 75141 Paris Cedex 03, France
}

(Reçu le 30 décembre 1976, accepté le 23 mai 1977)

\begin{abstract}
Résumé. - Nous décrivons les mesures de luminance spectrale énergétique entre 200 et $360 \mathrm{~nm}$ à l'aide d'un arc à hydrogène. Nous étudions particulièrement les diagnostics spectroscopiques qui permettent la détermination des paramètres du plasma. A partir de l'analyse des erreurs systématiques, nous déduisons une incertitude totale de $\pm 8 \%$ sur un étalonnage à $200 \mathrm{~nm}$. Enfin, nous donnons une brève description des sources de transfert.
\end{abstract}

\begin{abstract}
We describe spectral radiance measurements between 200 and $360 \mathrm{~nm}$ with a hydrogen arc as a standard source. We give special attention to the diagnostics that allow the determination of plasma parameters. From error analysis, we deduce an overall uncertainty of $\pm 8 \%$ at $200 \mathrm{~nm}$ on the calibration. We then review the principal transfer standards.
\end{abstract}

1. Introduction. - Les besoins de mesure d'énergie rayonnante dans le domaine ultraviolet se sont intensifiés depuis quelques années. Cela est dû, d'une part, à l'amélioration des techniques (techniques du vide, détection, source), d'autre part, au développement des recherches dans le domaine de la physique spatiale, de la physique des plasmas, de la biologie et des sciences de l'environnement. La méthode la plus directe pour mesurer un flux lumineux en unités énergétiques est de le comparer à une source étalon, c'est-à-dire à une source dont l'énergie rayonnée est calculable. Ainsi, on utilise dans le domaine spectral visible comme étalon le corps noir dont la luminance spectrale énergétique est prédite par la loi de Planck. Le maximum d'émission d'un corps noir est donné par la loi de Wien :

$$
\lambda_{\max } \times T=2885 \mu \mathrm{mK} .
$$

Ainsi, un corps noir ayant une température de $3000 \mathrm{~K}$ (le maximum réalisable), possède son maximum d'émission à $\lambda=1000 \mathrm{~nm}$. A $200 \mathrm{~nm}$ la luminance spectrale est de $7 \times 10^{4}$ fois plus faible. Le signal devient alors de l'ordre de grandeur de la lumière diffusée ce qui entraîne que le corps noir n'est pas adapté pour la plupart des applications comme source étalon dans l'ultraviolet. Les nombreuses recherches effectuées principalement dans les laboratoires de métrologie ont permis la mise au point de trois types de sources étalons pour le domaine ultraviolet :

$1^{\text {o }}$ la méthode des raies saturées [1],

$2^{\circ}$ le rayonnement synchrotron [2],

$3^{\circ}$ l'arc hydrogène $[3,4]$ dont nous décrivons dans

(*) Communication présentée au Congrès National de Physique des Plasmas, Paris, 6-10 décembre 1976. cet article les derniers développements à l'Institut National de Métrologie (I. N. M.).

2. Principe de l'expérience. - Le plasma d'hydrogène est créé dans un arc stabilisé. Cette source est utilisée depuis de nombreuses années pour les recherches de spectroscopie [5]. Ses principaux avantages sont, d'une part, sa stabilité et sa reproductibilité, d'autre part, la symétrie cylindrique qui permet d'observer le plasma axialement ou radialement. L'hydrogène a été choisi car ses probabilités de transition et ses facteurs de Gaunt sont exactement connus et permettent le calcul du rayonnement émis par le plasma. On utilise comme source étalon le fond continu émis par une colonne de plasma homogène entre 160 et $360 \mathrm{~nm}$. Dans cet intervalle spectral, le plasma est optiquement mince. On a donc :

$$
L(\lambda)=\varepsilon_{\lambda}\left(N_{\mathrm{e}}, T\right) \times l
$$

où :

$L(\lambda)$ est la luminance spectrale énergétique.

$\varepsilon_{\lambda}\left(N_{\mathrm{e}}, T\right)$ est le coefficient d'émission du plasma.

$l$ est sa longueur.

$N_{\mathrm{e}}$ et $T$ sont respectivement la densité électronique et la température.

Le calcul du coefficient d'émission [6] tient compte des phénomènes radiatifs suivants :

$\left.\begin{array}{lll}\text { (a) } \mathrm{H}^{+}+\mathrm{e}^{-} & \rightarrow \mathrm{H}(\mathrm{P})+\mathrm{h} v & \text { libre-lié } \\ \text { (b) } \mathrm{H}^{+}+\mathrm{e}^{-} & \rightarrow \mathrm{H}^{+}+\mathrm{e}^{-}+\mathrm{h} v & \text { libre-libre }\end{array}\right\} 93-\%$
$\left.\begin{array}{ll}\text { (c) } \mathrm{H}(\mathrm{P})+\mathrm{e}^{-} \rightarrow \mathrm{H}(\mathrm{q})+\mathrm{e}^{-}+\mathrm{h} v & \text { libre-libre } \\ \text { (d) } \mathrm{H}(\mathrm{P})+\mathrm{e}^{-} \rightarrow \mathrm{H}^{-}+\mathrm{hv} & \text { libre-lié }\end{array}\right\}$
$\left.\begin{array}{ll}\text { (e) } \mathrm{H}(\mathrm{P})+\mathrm{H}^{+} \rightarrow \mathrm{H}(\mathrm{q})+\mathrm{H}^{+}+\mathrm{h} v & \text { libre-libre } \\ \text { (f) } \mathrm{H}(\mathrm{P})+\mathrm{H}^{+} \rightarrow \mathrm{H}_{2}^{+}+\mathrm{h} v & \text { libre-lié }\end{array}\right\} 2 \%$ 
Les importances relatives des différents phénomènes sont calculées pour un plasma ayant une température de $15000 \mathrm{~K}$ entre 160 et $360 \mathrm{~nm}$. Les incertitudes liées au calcul du coefficient d'émission sont estimées à $\pm 2 \%$. Des vérifications expérimentales ont été faites dans le domaine visible et dans le domaine ultraviolet $[3,4]$. Aucun écart systématique n'a pu être décelé.

3. Montage expérimental (Fig. 1). - L'arc a un diamètre de $3 \mathrm{~mm}$ et une longueur de $32 \mathrm{~mm}$. On utilise un courant de $50 \mathrm{~A}$ stabilisé à $10^{-4}$ et on opère à pres-

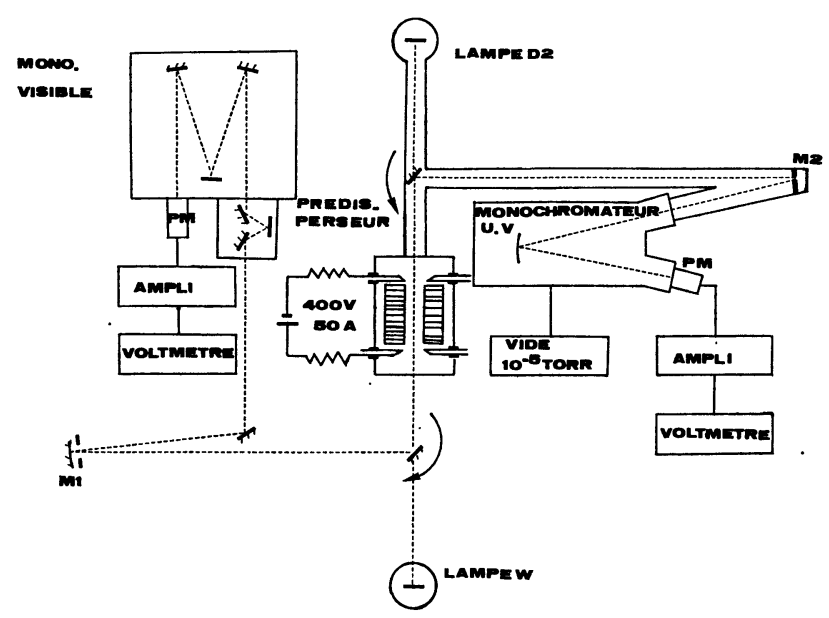

Fig. 1. - Montage expérimental.

[Experimental set-up.]

sion atmosphérique. Le plasma est observé axialement à ses deux extrémités. A l'une des extrémités, on effectue les mesures dans l'ultraviolet, à l'autre extrémité, on mesure la température et la densité électronique. On a vérifié préalablement que le plasma était symétrique. On focalise le centre du plasma sur la fente d'entrée du monochromateur. L'ouverture des faisceaux est de $\mathrm{F} / 200$, afin que la colonne de plasma visée soit la plus homogène possible. Les conditions optiques sont résumées dans le tableau I. Une expérience d'étalon-

\section{TABleaU I}

Conditions expérimentales

[Experimental conditions]

Focale

Visible

Miroir

$$
\overline{\mathrm{M}}_{1}
$$

Grandissement

Résolution spatiale

Monochromateur

$$
600 \mathrm{~mm}
$$

$$
1
$$

$0,1 \mathrm{~mm}$

$$
\begin{aligned}
& \frac{\mathrm{UV}}{\overline{\mathrm{M}}_{2}} \\
& 600 \mathrm{~mm} \\
& \frac{1}{3} \\
& 0,3 \mathrm{~mm}
\end{aligned}
$$

(1)

Czerny Turner Incidence norfocale : $\quad$ male focale :

$$
0,6 \mathrm{~m} \quad 1 \mathrm{~m}
$$

Résolution

Détecteur

$$
0,5 \mathrm{~nm}
$$

Photomultiplicateur

IP 28 nage consiste donc à mesurer simultanément les paramètres $N_{\mathrm{e}}$ et $T$ dans le domaine visible et le signal $S_{\mathrm{UV}}(\lambda)$ du plasma dans le domaine UV. Puis, on compare à travers le même dispositif optique $S_{\mathrm{UV}}(\lambda)$ au signal de la source à étalonner, soit $S(\lambda)$. La luminance spectrale de la source est donc :

$$
L(\lambda)=\varepsilon_{\lambda}\left(N_{\mathrm{e}}, T\right) \times l \times \frac{S(\lambda)}{S_{\mathrm{UV}}(\lambda)} .
$$

La figure 2 résume le processus. On déduit $N_{\mathrm{e}}$ et $T$ à l'aide de diagnostics spectroscopiques. Pour les

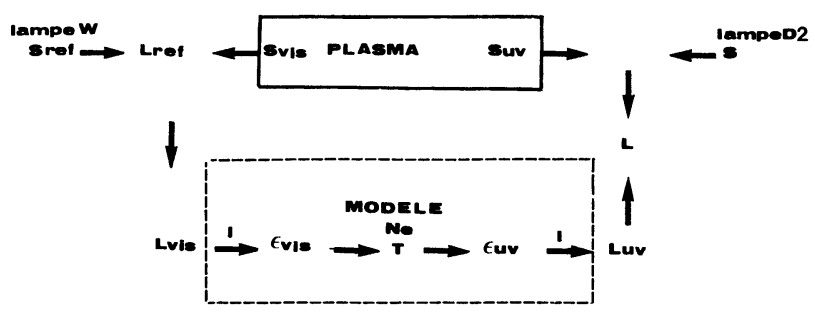

Fig. 2. - Procédure d'étalonnage.

[Calibration procedure.]

mesures absolues dans le domaine spectral visible, on utilise comme référence une lampe à ruban de tungstène étalonnée en luminance spectrale à partir d'un corps noir.

4. Diagnostics spectroscopiques et modèle. 4.1 ETAT D'ÉQUILIBRE DU PLASMA. - Du fait des densités élevées, le plasma est dominé par les processus collisionnels microréversibles qui vont imposer un état proche de l'équilibre thermodynamique local complet (ETLC). On a montré cependant [7] que deux phénomènes liés au niveau fondamental de l'atome peuvent perturber cet équilibre :

- la diffusion des neutres vers le centre de la décharge. Cette diffusion du fondamental est contrôlée par le phénomène d'échange de charge résonnant;

- la non-microréversibilité des collisions entre les électrons et le fondamental du fait de l'intervalle d'énergie important (proche de l'énergie d'ionisation) qui sépare les premiers états excités du fondamental. Un modèle de plasma réaliste doit donc tenir compte de ces deux effets. Si on suppose que les états excités sont en équilibre avec les électrons (hypothèse de 1'ETLP : équilibre thermodynamique local partiel), on peut alors écrire les équations suivantes :
$0,3 \mathrm{~nm}$

$$
N_{\mathrm{e}}=N_{\mathrm{i}}
$$

$\mathrm{P}=\left(N_{\mathrm{e}}+N_{\mathrm{i}}+N_{0}\right) k T$

$$
\text { quasi-neutralité }
$$

$\boldsymbol{\nabla} \cdot(N(1) \mathbf{V}(1))=\left[\frac{\partial N(1)}{\partial t}\right]_{\text {coll }}$

loi de Dalton

équation de conservation du fondamental

Photomultiplicateur Solar blind Be.0

$N_{0}=N(1)+\sum_{i} N(i)$ 
$\frac{N_{\mathrm{e}} N_{\mathrm{i}}}{N(j)}=\frac{2}{g_{j}}\left[\frac{2 \pi \mathrm{m}_{\mathrm{e}} k T}{h^{2}}\right]^{3 / 2}$

$\times \exp -\frac{\left(E_{\mathbf{i}}-E(j)-\Delta E_{\mathbf{i}}\right)}{k T}$

loi de Saha modifiée

$N_{\mathrm{i}}, N_{0}, N(j)$ sont, respectivement, les densités des protons, des neutres et du niveau $j$.

$E_{\mathrm{i}}, E(j)$ et $\Delta E_{\mathrm{i}}$ sont, respectivement, l'énergie d'ionisation, l'énergie du niveau $j$ et l'abaissement du potentiel d'ionisation.

L'équation (6) peut s'exprimer uniquement en fonction de la température et des densités [7]. On a donc $5+j$ inconnues $\left(N_{\mathrm{e}}, N_{\mathrm{i}}, N_{0}, N(1), N(j), T\right)$ pour $4+j$ équations. Il suffit par conséquent d'un diagnostic spectroscopique pour déterminer le système.

4.2 Diagnostics SPECTROSCOPIQUES DANS LE DOMAINE VISIBLE. - 4.2.1 Mesure de la luminance d'une raie

$$
\varepsilon_{j k}=\frac{h c}{4 \pi \lambda} A_{j k} N(j)
$$

La parfaite connaissance des probabilités de transition $A_{j k}$ de l'hydrogène permet de déduire $N(j)$ avec, comme seule erreur, l'erreur sur la mesure. Seules les raies $\mathrm{H}_{\beta}(j=4)$ et $\mathrm{H}_{\gamma}(j=5)$ peuvent être utilisées. $\mathbf{H}_{\alpha}(j=3)$ est autoabsorbée et les raies issues des transitions supérieures sont noyées dans le fond continu du fait de l'abaissement du potentiel d'ionisation.

4.2.2 Mesure de la luminance spectrale du fond continu. - On mesure le fond continu entre 500 et $600 \mathrm{~nm}$. Le système (2), (4), (5), (6), (7) et (8) permet de déterminer les paramètres du plasma par itérations. Il faut tenir compte dans le calcul du coefficient d'émission du fond continu de l'importance des ailes des raies.

4.2.3 Mesure du profil des raies. - La mesure des élargissements Stark des raies $\mathrm{H}_{\beta}$ et $\mathrm{H}_{\gamma}$ permet de déduire directement la densité électronique à partir des travaux récents de Wiese, Kelleher et Paquette [8].

4.2.4 Mesure du rapport de l'intensité $d u$ fond continu sur l'intensité d'une raie. - Le rapport $\varepsilon_{\lambda} / \varepsilon_{j k}$ est proportionnel à $N_{\mathrm{e}} N_{\mathrm{i}} / N(j)$ à condition que les processus (c) et (d) du paragraphe 2 soient négligeables. Cette condition est assez bien réalisée en utilisant le

\section{TABLEAU II}

\section{Résultats expérimentaux et écarts types sur vingt expériences}

[Experimental results and standard deviations for 20 runs]

\begin{tabular}{lccccc} 
& $\mathbf{H}_{\boldsymbol{\beta}}$ & $\mathbf{H}_{\gamma}$ & \multicolumn{1}{c}{$\mathrm{C}_{\text {on }}$} & $\Delta \mathbf{H}_{\boldsymbol{\beta}}$ & \multicolumn{1}{c}{$\Delta \mathbf{H}_{\gamma}$} \\
& - & - & - & - & - \\
$N_{\mathrm{e}}$ & 1,14 & 1,10 & 1,10 & 1,14 & 1,02 \\
$\times 10^{-17} \mathrm{~cm}^{-3}$ & $\pm 0,05$ & $\pm 0,05$ & $\pm 0,05$ & $\pm 0,06$ & $\pm 0,04$ \\
$T_{\mathrm{e}}(\mathrm{K})$ & 14700 & 14570 & 14600 & 14700 & 14300
\end{tabular}

fond continu émis dans le proche UV (300-350 nm). On obtient directement la température électronique du plasma.

4.2.5 Résultats. - Les moyennes des résultats expérimentaux sont reportées dans le tableau II. On constate un bon accord entre toutes les mesures, excepté la mesure du profil de $\mathrm{H}_{\gamma}$. La cause de cette différence n'est pas connue.

5. Analyse des principales erreurs systématiques liées à l'étalonnage d'une source. - La luminance spectrale énergétique d'une source est calculée à l'aide de la formule (3). La procédure (Fig. 2) consiste à opérer un transfert d'information du domaine visible au domaine UV. Nous devons donc distinguer deux types d'erreurs :

- les erreurs directes qui agissent directement sur la grandeur à mesurer (par exemple, l'erreur systématique sur la luminance spectrale de la lampe à ruban de tungstène);

- les erreurs de transfert qui interviennent dans le passage de la luminance visible $\left(L_{\text {vis }}\right)$ à la luminance UV $\left(L_{\text {uv }}\right)$ (par exemple, la longueur du plasma).

A l'inverse des erreurs directes, certaines erreurs de transfert se compensent partiellement. Par exemple, une erreur de $5 \%$ sur la longueur du plasma entraîne une erreur de $1 \%$ sur $L_{\text {uv }}$. Les principales erreurs systématiques sont mentionnées dans le tableau III. En combinant ces erreurs quadratiquement, on arrive à une incertitude totale à $200 \mathrm{~nm}$ :

$$
\frac{\Delta L_{\mathrm{uv}}}{L_{\mathrm{uv}}}= \pm 8 \% \text {. }
$$

\section{TABLEAU III}

Les erreurs systématiques

[Systematic errors]

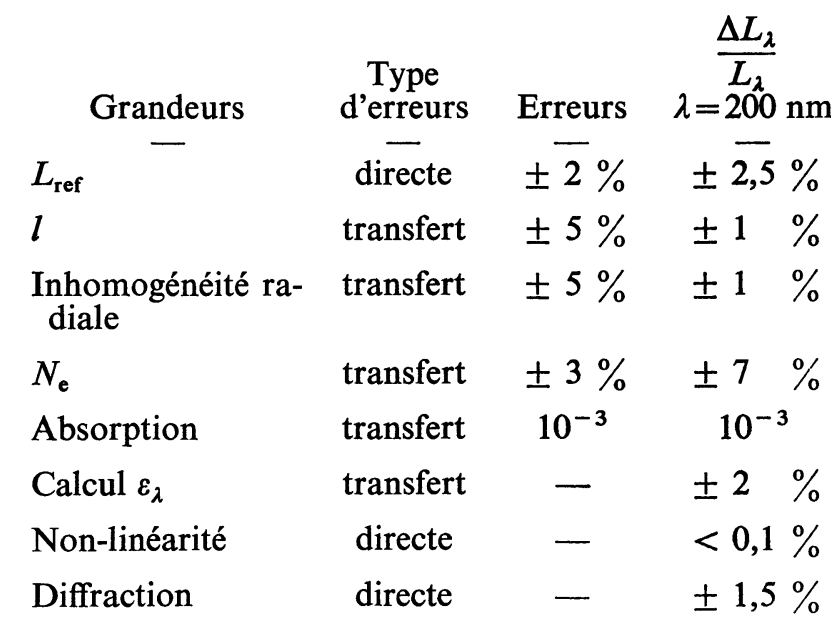

6. Les étalons de transfert. - Les sources de transfert utilisées actuellement sont des lampes à deutérium qui sont commercialisées par la plupart des fabricants. 
Ces lampes qui fonctionnent sous un courant continu de $300 \mathrm{~mA}$ et sous une tension de $100 \mathrm{~V}$ fournissent un spectre continu entre 165 et $360 \mathrm{~nm}$. L'avantage de ces sources est leur faible coût et leur maniabilité. L'inconvénient majeur est le vieillissement qui varie d'une lampe à l'autre et qui nécessite des comparaisons fréquentes par rapport à l'étalon primaire. La figure 3 montre un exemple d'une courbe d'étalonnage. Ces

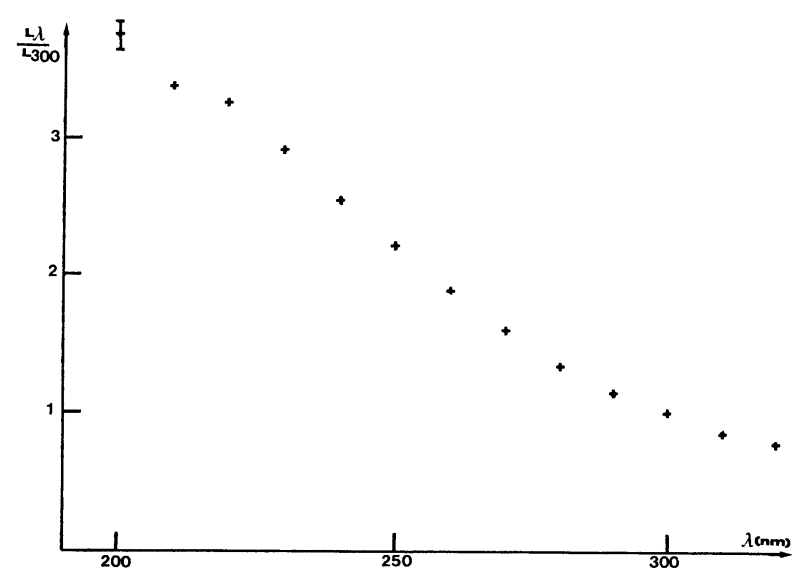

Fig. 3. - Exemple d'étalonnage d'une lampe à deutérium. $L_{300}=9,46 \mu \mathrm{W} . \mathrm{mm}^{-2} \cdot \mathrm{nm}^{-1}$. str. $\pm 5 \%$.

[Calibration of a D2 lamp.

$$
L_{300}=9.46 \mu \mathrm{W} \mathrm{mm}-2 \mathrm{~nm}^{-1} \mathrm{st}^{-1} \pm 5 \% \text {.] }
$$

résultats ont été obtenus sans utiliser le système de pompage ce qui limite la longueur d'onde à $200 \mathrm{~nm}$. Cette courbe n'est évidemment valable que pour des utilisations de la source aussi proches que possible de l'étalonnage par le laboratoire primaire. On étudie actuellement au National Bureau of Standards (N. B. S.) et à l'I. N. M. une nouvelle source de transfert constituée par un arc à argon qui permet de s'affranchir du vieillissement et d'étendre le domaine spectral.

7. Conclusion. - Afin d'améliorer et d'uniformiser les techniques d'étalonnage dans le domaine ultraviolet, l'Institut National de Métrologie poursuit un double objectif :

- Le premier est de rendre l'étalonnage crédible. Pour cela, nous organisons des comparaisons avec tous les laboratoires intéressés. Actuellement, une telle comparaison est en cours avec le National Physical Laboratory (N. P. L.), en Grande-Bretagne. Les résultats seront prochainement publiés. Nous avons également entrepris une expérience de comparaison entre le rayonnement synchrotron fourni par l'anneau de collision d'Orsay (A. C. O. LURE) et l'arc hydrogène. Enfin, nous poursuivons une collaboration scientifique très étroite avec le National Bureau of Standards.

- Le deuxième objectif est de minimiser la perte de précision d'un étalonnage entre le laboratoire primaire et l'utilisateur. Pour cela, il faut que la source de transfert soit la mieux adaptée possible aux besoins de l'utilisateur, que le laboratoire primaire fournisse un mode d'emploi détaillé et, enfin, assure un contrôle régulier.

Remerciements. - Ce travail a été incité par la Direction des Recherches et Moyens d'Essais (D. R. M. E.).

\section{Bibliographie}

[1] BoLdt, G., Space Sci. Rev. 11 (1970) 728.

[2] GROUPE LURE et al., L'utilisation du rayonnement synchrotron en France, Ann. Phys. 9 (1975) 1.

[3] Ott, W. R., FiffFe-Prevost, P., Wiese, W. L., Appl. Opt. 12 (1973) 1618.

[4] Ott, W. R., Behringer, K., Gieres G., Appl. Opt. 14 (1975) 2121.
[5] Olsen, H. N., J. Quant. Spect. Radia. Trans. 3 (1963) 305.

[6] Roberts, J. R., Voigt, P. A., J. Res. Nat. Bur. Stand. 75A (1971) 291.

[7] Fieffe-Prevost, P., Phys. of ionized Gases, Dubrovnik, SPIG (76), (J. Stefan Institute, Ljubljana), 1976.

[8] Wiese, W. L., Kelleher, D. E., Paquette, D. R., Phys. Rev. A6 (1972) 1132. 